\title{
Fourier Transform Infrared Radiation Spectroscopy Applied for Wood Rot Decay and Mould Fungi Growth Detection
}

\author{
Bjørn Petter Jelle $e^{1,2}$ and Per Jostein Hovde ${ }^{2}$ \\ ${ }^{1}$ SINTEF Building and Infrastructure, Department of Materials and Structures, 7465 Trondheim, Norway \\ ${ }^{2}$ Department of Civil and Transport Engineering, Norwegian University of Science and Technology (NTNU), \\ 7491 Trondheim, Norway
}

Correspondence should be addressed to Bjørn Petter Jelle, bjorn.petter.jelle@sintef.no

Received 14 March 2012; Revised 29 May 2012; Accepted 30 May 2012

Academic Editor: John W. Gillespie

Copyright (C) 2012 B. P. Jelle and P. J. Hovde. This is an open access article distributed under the Creative Commons Attribution License, which permits unrestricted use, distribution, and reproduction in any medium, provided the original work is properly cited.

Material characterization may be carried out by the attenuated total reflectance (ATR) Fourier transform infrared (FTIR) radiation spectroscopical technique, which represents a powerful experimental tool. The ATR technique may be applied on both solid state materials, liquids, and gases with none or only minor sample preparations, also including materials which are nontransparent to IR radiation. This facilitation is made possible by pressing the sample directly onto various crystals, for example, diamond, with high refractive indices, in a special reflectance setup. Thus ATR saves time and enables the study of materials in a pristine condition, that is, the comprehensive sample preparation by pressing thin $\mathrm{KBr}$ pellets in traditional FTIR transmittance spectroscopy is hence avoided. Materials and their ageing processes, both ageing by natural and accelerated climate exposure, decomposition and formation of chemical bonds and products, may be studied in an ATR-FTIR analysis. In this work, the ATR-FTIR technique is utilized to detect wood rot decay and mould fungi growth on various building material substrates. An experimental challenge and aim is to be able to detect the wood rot decay and mould fungi growth at early stages when it is barely visible to the naked eye. Another goal is to be able to distinguish between various species of fungi and wood rot.

\section{Introduction}

Wood rot decay and mould fungi growth on building materials represent a challenging problem. In addition to the building damages, for example, wood rot decay, often due to unwanted water ingress, one of the large risks is related to mould fungi growth. Trapped water or moisture, for example, between watertight barriers, which has no possible way to dry or drain out, may lead to mould fungi growth under certain conditions. Unfortunately, these conditions are often fulfilled. The mould fungi initiation and growth are dependent upon the following factors:

(i) availability of fungal spores, which are almost always present, except under sterile conditions,

(ii) nourishment, for example, wood,

(iii) moisture,

(iv) temperature, (v) oxygen,

(vi) time.

Many different species of mould fungi exist, each of them with various requirements to the above initiation and growth factors. For example, a specific fungal specimen may be latent with no growth below a certain temperature and might be exterminated above a certain temperature, where the temperature range will vary for each specimen. The exact relationship between mould fungi growth, with release of airborne fungi, and health problems is not yet fully understood. For further information on this topic see, for example, the work by Holme et al. [1, 2]. Nevertheless, that there is a health risk is not questioned, and many people have become sick due to moisture entrapment and the subsequent fungi growth. Occasionally, it has been necessary to evacuate buildings infected with mould fungi. Further information concerning air quality, moisture, and related issues may be 
found in EPA [3], IEE [4], Harriman III and Lstiburek [5], Lstiburek [6], The National Academies Press [7], and WHO [8].

In this work, we are trying to utilize the attenuated total reflectance (ATR) Fourier transform infrared (FTIR) radiation experimental technique in order to detect wood rot decay and mould fungi growth on building material substrates. The ATR-FTIR technique makes it possible to study materials which are nontransparent to IR radiation in a pristine condition. That is, the extensive, timeconsuming, and often cumbersome sample preparation by pressing thin $\mathrm{KBr}$ pellets as in traditional FTIR transmittance spectroscopy, which might even change the sample material in question, is avoided. The ATR technique is based on a special reflectance setup where the sample material is pressed directly onto various crystals with high refractive indices, for example, diamond.

An experimental challenge of special interest is to be able to detect the wood rot decay and fungi growth at early stages when the fungi species are barely visible to the naked eye. Ultimately, one may envision future hand-held instruments scanning over a larger area and being able to detect wood rot decay or mould fungi growth before it is visible. The task is experimentally challenging, both to differentiate the biological growth and decay impact on the attacked material from the substrate material itself, as well as to be able to distinguish between various species of fungi and wood rot. Popularized, one may say that you are becoming what you are eating, that is, with respect to FTIR spectra differentiation between the wood rot and mould fungi products versus the substrate material. Several studies are carried out applying infrared spectroscopy in various studies of microorganisms, for example, by Fischer et al. [9], Humar et al. [10], Irudayaraj et al. [11], Kos et al. [12], Mohebby [13], Naumann et al. [14], Ngo-Thi et al. [15], Orsini et al. [16], Pandey and Pitman [17, 18], and Wenning et al. [19]. Furthermore, Humar et al. [10], Kos et al. [12], Mohebby [13], and Orsini et al. [16] performed parts of their studies by utilizing the ATR-FTIR spectroscopical technique, describing a promising method with many advantages in these types of investigations. For application of the ATR-FTIR method for climate-aged wood substrates and for comparison of accelerated climate ageing methods of polymer building materials by FTIR, it is referred to the studies by Jelle et al. [20] and Jelle and Nilsen [21], respectively.

\section{Experimental}

2.1. Sample Materials. The small sample materials for the FTIR measurements have been collected from the larger field samples depicted in Figure 1, with the following sample categories.

(i) Mould fungi on GU plaster board (GU = exterior grade gypsum board).

(a) Unexposed GU plaster board.

(b) Exposed GU plaster board with mould fungi scraped off, that is, measured on plaster board. (c) Mould fungi on GU plaster board.

(d) Mould fungi scraped off from GU plaster board, that is, measured on a small mould fungi powder pellet.

(ii) Mould fungi on wood.

(a) Unexposed wood (30 $\mathrm{mm}$ below surface), the wood piece is actually an exposed sample, but the measured sample is taken $30 \mathrm{~mm}$ below the outer surface of the wood piece, where there are no signs of mould fungi.

(b) Exposed wood ( $2 \mathrm{~mm}$ below surface), some mould fungi may be present $2 \mathrm{~mm}$ below the outer surface.

(c) Mould fungi on wood.

(d) Mould fungi scraped off from wood, that is, measured on a small mould fungi powder pellet.

(iii) Wood with wood rot decay.

(a) Unexposed wood ( $30 \mathrm{~mm}$ below surface), the wood piece is actually an exposed sample, but the measured sample is taken $30 \mathrm{~mm}$ below the outer surface of the wood piece, where there are no signs of wood rot decay.

(b) Exposed wood ( $2 \mathrm{~mm}$ below surface), some wood rot decay may be present $2 \mathrm{~mm}$ below the outer surface.

(c) Wood surface damaged by wood rot, measured sample close to wood rot on wood, that is, adjacent to (but not onto) wood surface heavily infected with wood rot.

(d) Wood rot on wood, area on wood piece heavily infected with wood rot.

(e) Wood rot scraped off from wood, that is, measured on a small wood rot powder pellet.

(iv) Reference materials (for check/comparison of FTIR absorbance peaks).

(a) Water, distilled.

(b) Gypsum, nondried.

(c) Gypsum, dried.

2.2. FTIR Measurements. The FTIR material characterization was carried out with a Thermo Nicolet 8700 FTIR spectrometer with a Smart Orbit accessory, that is, a horizontal attenuated total reflectance (ATR) accessory (single reflection) with a diamond crystal, in the wavelength range $4000 \mathrm{~cm}^{-1}(2.5 \mu \mathrm{m})$ to $400 \mathrm{~cm}^{-1}(25 \mu \mathrm{m})$ in an atmosphere with minimalized $\mathrm{CO}_{2}$ and $\mathrm{H}_{2} \mathrm{O}$ content through purging by a Parker Balston 74-5041 FTIR Purge Gas Generator.

Each FTIR spectrum presented is based on a recording of 32 scans at a resolution of $4 \mathrm{~cm}^{-1}$. In order to ensure satisfactory contact between the ATR diamond crystal and the sample, three or more FTIR spectra were recorded at various 


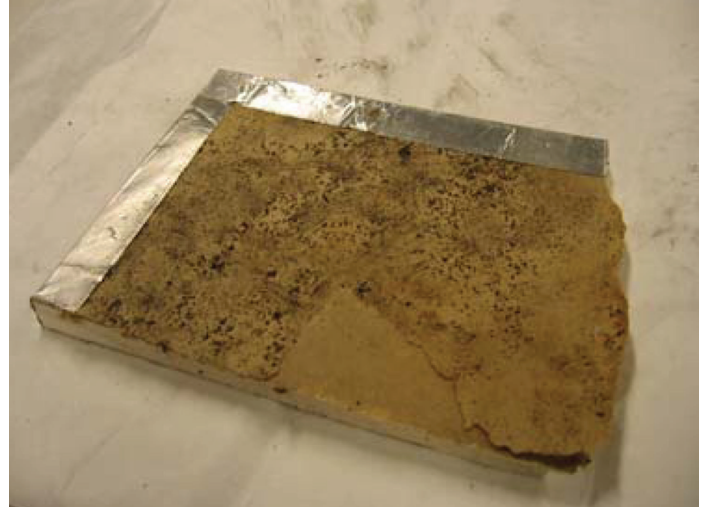

(a)

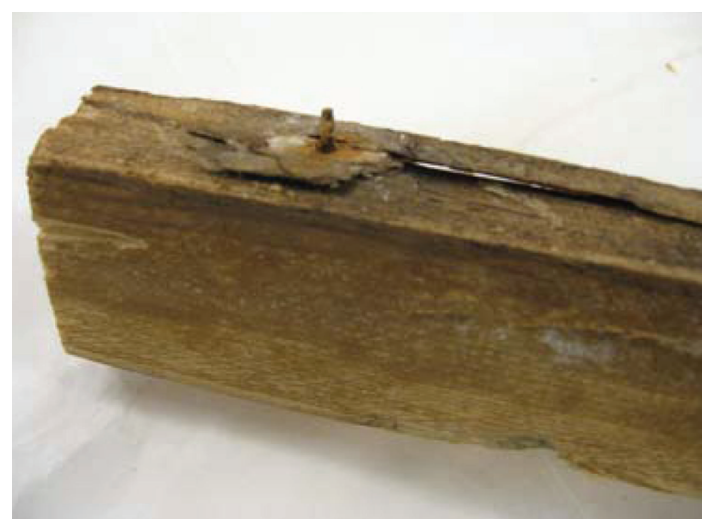

(b)

FIGURE 1: Large field samples from where smaller samples have been collected for the FTIR measurements. Mould fungi on GU plaster board (a) and mould fungi on wood and wood rot (b). GU: exterior grade gypsum board ("outdoor use").

locations on the sample. Relatively hard surfaces complicate accurate quantitative measurements (height of absorbance peaks) due to varying contact with the ATR crystal for the different samples. Air between sample and ATR crystal results in a weaker absorbance signal. Unless other conditions indicate otherwise (e.g., inhomogeneities, impurities, etc.), the FTIR spectra with the largest absorbance peaks represent the most correct measurements on one and the same sample with equal ageing time, and hence these spectra are chosen as they are assumed to be the most correct ones. Qualitative measurements (location of absorbance peaks at wave numbers) do not represent a problem as long as the contact area is large enough to ensure a sufficient strong measurement signal.

The FTIR spectra given in this work have not been ATR corrected, neither with respect to penetration depths nor absorbance band shifts, which both are dependent on the refractive indices of the sample and the ATR crystal (diamond in this case) and the angle of incident radiation. The penetration depth is in addition also dependent on the radiation wavelength and increases with increasing wavelength (decreasing wave number). That is, noncorrected ATR spectra have much stronger absorbance bands at longer wavelengths (smaller wave numbers) than at shorter wavelengths (larger wave numbers). Note that it should always be stated if an ATR-FTIR spectrum has been ATR corrected or not, because it is important during computerized database spectra comparison searches. As we in this work are solely comparing the ATR-FTIR spectra measured within this work, there is no need for performing any ATR corrections. Besides, the raw ATR-FTIR data in either transmittance or logarithmic absorbance mode are usually preferred. It should also be noted that often one does not know the refractive indices of the samples, which are measured, thus errors might be introduced in the ATR-corrected spectra as the refractive index of the sample is an input parameter in the ATR correction.

The FTIR measurements were performed on several samples within each sample category as described in the sample materials section above, of which only one single representative spectrum for each sample category is shown in the following results and discussion section.

\section{Results and Discussion}

FTIR absorbance spectra versus wave number between 4000 $400 \mathrm{~cm}^{-1}$ for the various mould fungi and wood rot samples are presented in Figures 2-6. Mould fungi samples at various exposure degrees on GU plaster board are shown in Figure 2. Mould fungi samples at different exposure levels on wood are given in Figure 3. Wood rot samples at various decay levels are shown in Figure 4. Figure 5 gives the FTIR absorbance versus wave number between $4000-400 \mathrm{~cm}^{-1}$ for mould fungi on GU plaster board, mould fungi on a wood sample, and wood rot at selected exposure levels (from Figures 2-4). In addition, the water spectrum is also plotted as a comparison. Figure 6 shows the FTIR absorbance versus wave number between $1800-1400 \mathrm{~cm}^{-1}$ for mould fungi on GU plaster board, mould fungi on a wood sample, and wood rot at selected exposure levels (from Figures 2-5). In addition, the water, nondried gypsum, and dried gypsum spectra are also plotted as a comparison.

As the absorption of electromagnetic radiation, for example, IR radiation, follows the Beer-Lambert law, that is, the radiation is decreasing exponentially with the penetration depth in the actual material, it is often helpful to plot the spectra on a logarithmic absorbance scale versus wavelength. Hence, a representative spectrum is chosen from each of the samples and plotted on a logarithmic absorbance scale for quantitative studies. Mathematically and physically, it follows that a doubling of the logarithmic absorbance, also called optical density, is interpreted as a doubling of material thickness or a doubling of concentration of absorption active agents.

The mould fungi growth and wood rot decay makes the sample material undergo chemical changes and may then as a result of the chemical reactions change the actual thickness of the sample. In this work, the experiments are conducted by applying the ATR equipment with the FTIR spectrometer. Hence, the IR radiation is only penetrating into a thin surface layer of the actual sample. Thus, with respect to the 


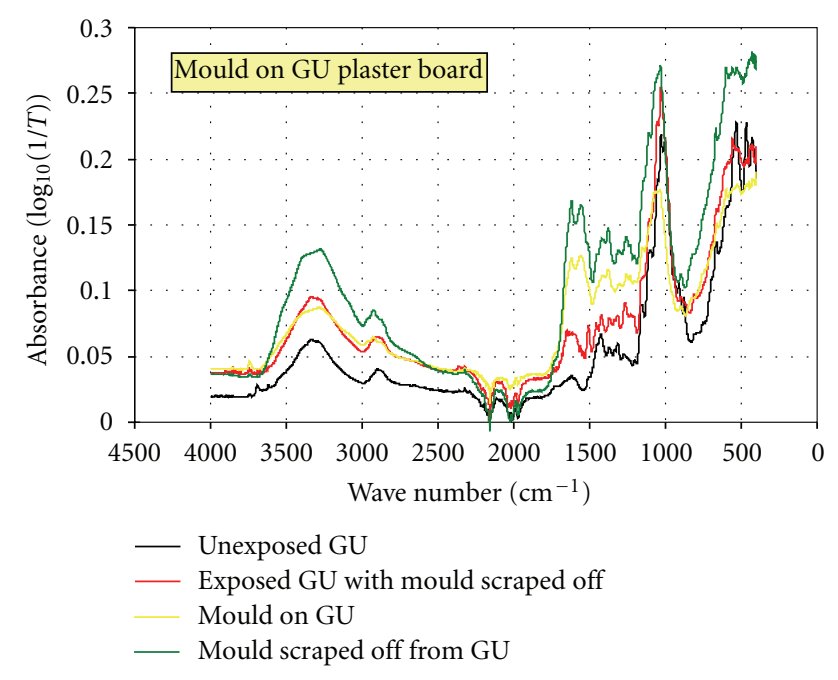

Figure 2: Absorbance (logarithmic) versus wave number between $4000-400 \mathrm{~cm}^{-1}$ for mould fungi at various exposure levels on GU plaster board. ( $T$ : transmittance).

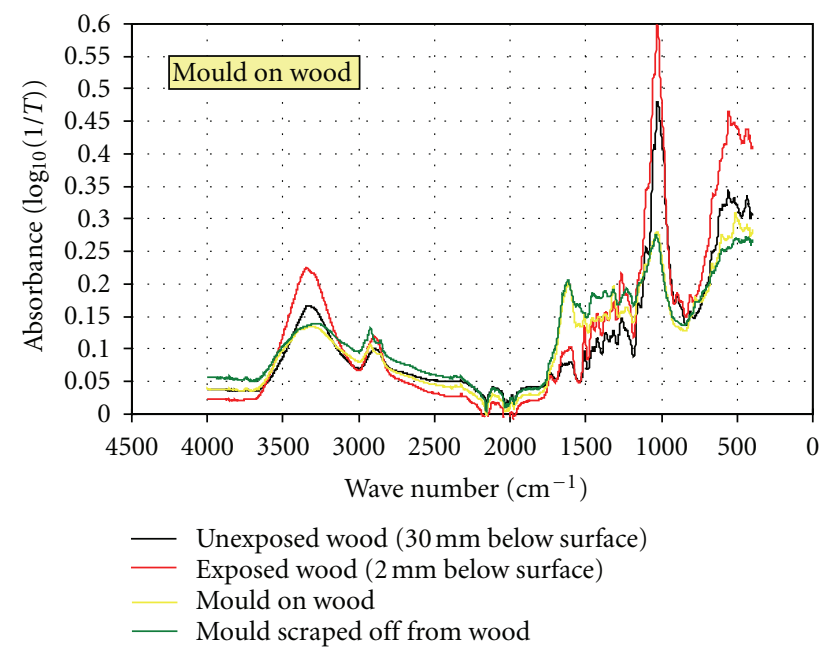

FIGURE 3: Absorbance (logarithmic) versus wave number between $4000-400 \mathrm{~cm}^{-1}$ for mould fungi at various exposure levels on a wood sample.

experiments carried out in this work, the material thickness will then be regarded as approximately constant, that is, the change in the IR absorbance is explained by an increase or decrease of absorption active agents within the sample material undergoing the chemical transformation.

In Figure 6, the large ellipse encircles FTIR absorbance peaks between $1630 \mathrm{~cm}^{-1}$ and $1580 \mathrm{~cm}^{-1}$, which may be related to mould fungi and/or wood rot, but this is more uncertain due to one of the water peaks $\left(1635 \mathrm{~cm}^{-1}\right)$ close to this location, which may cause quantitative changes or some smaller (qualitative) wave number shifts. Note that any microbiological growth may increase the water content. Furthermore, in order to quantify any potential pollution (error)

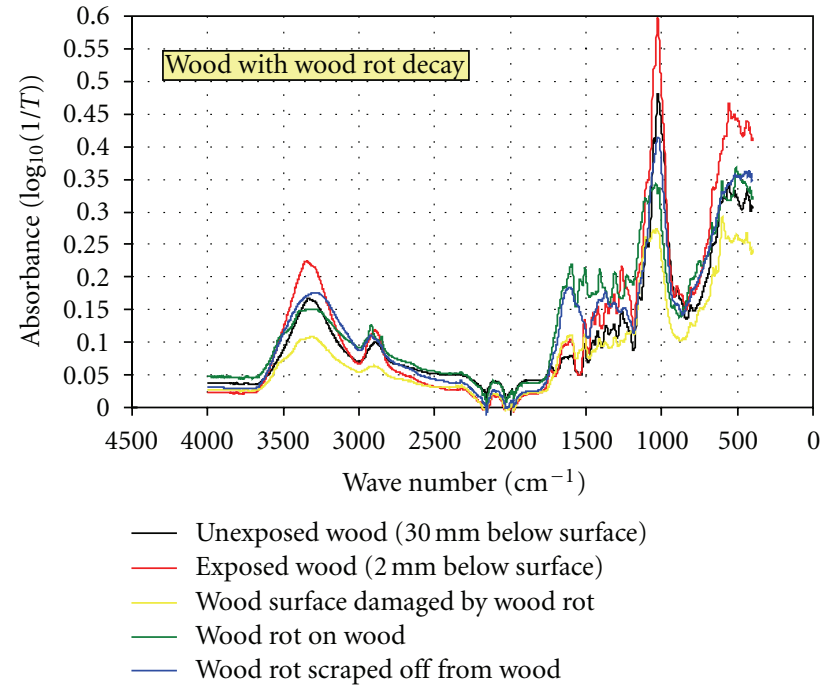

Figure 4: Absorbance (logarithmic) versus wave number between $4000-400 \mathrm{~cm}^{-1}$ for wood rot at various exposure levels of wood rot decay.

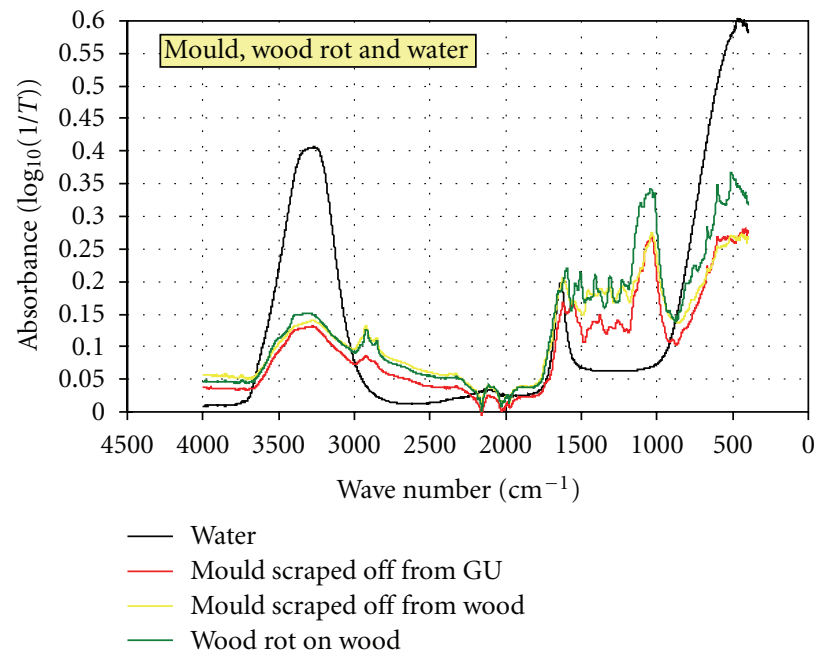

FIGURE 5: Absorbance (logarithmic) versus wave number between $4000-400 \mathrm{~cm}^{-1}$ for mould fungi on GU plaster board, mould fungi on a wood sample and wood rot at selected exposure levels. In addition, the water spectrum is also plotted as a comparison. From Figures 2-4 (except water spectrum).

from any astray gypsum powder, the gypsum peaks are also depicted, as these falls in the same wave number range. Also note that different adjacent neighbour atoms in a compound, for example, in a polymer chain, will shift the wave number somewhat for the absorbance peak corresponding to the chemical bond in question. The small ellipse in Figure 6 encircles FTIR absorbance peaks, which are found to be characteristic for the mould fungi and wood rot. These peaks, also including the other samples depicted in Figures 2-4, are located between $1558 \mathrm{~cm}^{-1}$ and $1535 \mathrm{~cm}^{-1}$. That is, the chemical bonds, which are the source for the absorbance peaks at these wave numbers, are found to be present and characteristic for the various mould fungi and wood rot 


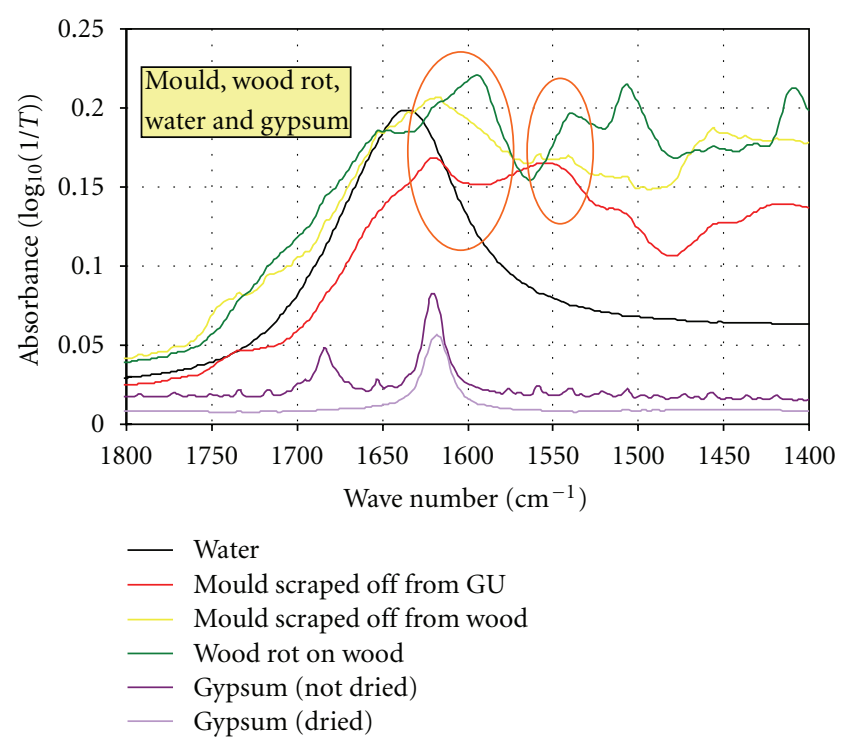

FIGURE 6: Absorbance (logarithmic) versus wave number between $1800-1400 \mathrm{~cm}^{-1}$ for mould fungi on GU plaster board, mould fungi on a wood sample, and wood rot at selected exposure levels. In addition, the water, nondried gypsum and dried gypsum spectra are also plotted as a comparison. From Figure 5 (except gypsum spectra). See discussion in text about the FTIR absorbance peaks within the large (left) and small (right) ellipse encircling the absorbance peaks.

products studied in this work. The nature and origin of these absorbance peaks linked to specific chemical bonds are not currently known by the authors.

Note that the moisture condition of the samples, that is, how much water they contain, will influence the FTIR spectra. Therefore, the water absorbance peaks are depicted in Figures 5 and 6. Water has two absorbance peaks, a broad peak around $3300 \mathrm{~cm}^{-1}$ and a sharper peak around $1635 \mathrm{~cm}^{-1}$ (pure water, not counting any impurities or volatile compounds). However, as long as the moisture does not induce any chemical reactions and hence no chemical bond changes, the moisture content in the various materials will not cause the appearance or disappearance of any absorbance peaks, that is, ideally no qualitative changes. Nevertheless, close to the water absorbance peaks, quantitative changes may occur. Naturally, as stated in the introduction section, moisture is one of the factors the mould fungi initiation and growth are dependent upon, and moisture may, therefore, ultimately still be regarded as one of the factors for initially causing changes to a material substrate and hence the respective FTIR spectra. Furthermore, also with respect to the moisture and water content in the samples, the purpose of this study was to use various real-life field samples to attempt to detect wood rot decay and mould fungi growth on building materials. That is, although also important and may be the subject in future studies, the purpose in this study was not to cultivate and study ideal or simple monoculture species.

Hence, in this study it has been demonstrated that the ATR-FTIR spectroscopical technique may be applied in order to detect wood rot decay and mould fungi growth on building materials collected as real-life field samples. Further studies and experimental investigations are needed in order to elaborate the suitability, reliability, limitations, and future potential possibilities of the future applications of this method. Furthermore, in future applications of this experimental method as a detection tool capable of differentiating between various wood rot and mould fungi species, there is a need to build up large reference databases containing FTIR spectra of the different species.

\section{Conclusions}

This study has demonstrated that the attenuated total reflectance (ATR) Fourier transform infrared (FTIR) spectroscopical technique may be applied in order to detect wood rot decay and mould fungi growth on building materials collected as real-life field samples. Nevertheless, further studies and experimental investigations are needed in order to elaborate the suitability, reliability, limitations, and potential possibilities of the future applications of this method.

\section{Acknowledgments}

This work has been supported by the Research Council of Norway, AF Gruppen, Glava, Hunton Fiber AS, Icopal, Isola, Jackon, maxit, Moelven ByggModul, Rambøll, Skanska, Statsbygg, and Takprodusentenes Forskningsgruppe through the SINTEF and NTNU research project "Robust Envelope Construction Details for Buildings of the 21st Century" (ROBUST).

\section{References}

[1] J. Holme, S. Geving, and J. A. Jenssen, "Moisture and mould damage in norwegian houses," in Proceedings of the 8th Symposium on Building Physics in the Nordic Countries, C. Rode, Ed., pp. 1213-1220, Danish Society of Engineers, Copenhagen, Denmark, June, 2008.

[2] J. Holme, L. Hägerhed-Engman, J. Mattsson, J. Sundell, and C.-G. Bornehag, "Culturable mold in indoor air and its association with moisture-related problems and asthma and allergy among Swedish children," Indoor Air, vol. 20, no. 4, pp. 329-340, 2010.

[3] EPA, “The inside story: a guide to indoor air quality," United States Environmental Protection Agency, United States Consumer Product Safety Commission, Office of Radiation and Indoor Air, EPA Document 402-K-93-007, 1995.

[4] IEE, Guidelines for Good Indoor Air Quality in Office Premises, Institute of Environmental Epidemiology, Ministry of the Environment, Singapore, 1st edition, 1996.

[5] L. G. Harriman III and J. W. Lstiburek, The ASHRAE Guide for Buildings in Hot \& Humid Climates, Refrigerating and AirConditioning Engineers, American Society of Heating, 2nd edition, 2009.

[6] J. Lstiburek, Investigating and Diagnosing Moisture Problems, vol. 108 of Building Science Digest, Building Science Press, 2006. 
[7] The National Academies Press, Damp Indoor Spaces and Health, Institute of Medicine of the National Academies, The National Academies Press, Washington, DC, USA, 2004.

[8] WHO, WHO Guidelines for Indoor Air Quality: Dampness and Mould, World Health Organization, Copenhagen, Denmark, 2009.

[9] G. Fischer, S. Braun, R. Thissen, and W. Dott, "FT-IR spectroscopy as a tool for rapid identification and intra-species characterization of airborne filamentous fungi," Journal of Microbiological Methods, vol. 64, no. 1, pp. 63-77, 2006.

[10] M. Humar, B. Bučar, and F. Pohleven, "Brown-rot decay of copper-impregnated wood," International Biodeterioration and Biodegradation, vol. 58, no. 1, pp. 9-14, 2006.

[11] J. Irudayaraj, H. Yang, and S. Sakhamuri, "Differentiation and detection of microorganisms using fourier transform infrared photoacoustic spectroscopy," Journal of Molecular Structure, vol. 606, no. 1-3, pp. 181-188, 2002.

[12] G. Kos, H. Lohninger, and R. Krska, "Fourier transform midinfrared spectroscopy with attenuated total reflection (FTIR/ATR) as a tool for the detection of Fusarium fungi on maize," Vibrational Spectroscopy, vol. 29, no. 1-2, pp. 115-119, 2002.

[13] B. Mohebby, "Attenuated total reflection infrared spectroscopy of white-rot decayed beech wood," International Biodeterioration and Biodegradation, vol. 55, no. 4, pp. 247-251, 2005.

[14] A. Naumann, M. Navarro-González, S. Peddireddi, U. Kües, and A. Polle, "Fourier transform infrared microscopy and imaging: detection of fungi in wood," Fungal Genetics and Biology, vol. 42, no. 10, pp. 829-835, 2005.

[15] N. A. Ngo-Thi, C. Kirschner, and D. Naumann, "Characterization and identification of microorganisms by FT-IR microspectrometry," Journal of Molecular Structure, vol. 661662, pp. 371-380, 2003.

[16] F. Orsini, D. Ami, A. M. Villa, G. Sala, M. G. Bellotti, and S. M. Doglia, "FT-IR microspectroscopy for microbiological studies," Journal of Microbiological Methods, vol. 42, no. 1, pp. 17-27, 2000.

[17] K. K. Pandey and A. J. Pitman, "FTIR studies of the changes in wood chemistry following decay by brown-rot and white-rot fungi," International Biodeterioration and Biodegradation, vol. 52, no. 3, pp. 151-160, 2003.

[18] K. K. Pandey and A. J. Pitman, "Examination of the lignin content in a softwood and a hardwood decayed by a brown-rot fungus with the acetyl bromide method and Fourier transform infrared spectroscopy," Journal of Polymer Science A, vol. 42, no. 10, pp. 2340-2346, 2004.

[19] M. Wenning, H. Seiler, and S. Scherer, "Fourier-transform infrared microspectroscopy, a novel and rapid tool for identification of yeasts," Applied and Environmental Microbiology, vol. 68, no. 10, pp. 4717-4721, 2002.

[20] B. P. Jelle, P. Rüther, and P. J. Hovde, "Investigations of accelerated climate aged wood substrates by Fourier transform infrared material characterization," Advances in Materials Science and Engineering. In press.

[21] B. P. Jelle and T. N. Nilsen, "Comparison of accelerated climate ageing methods of polymer building materials by attenuated total reflectance Fourier transform infrared radiation spectroscopy," Construction and Building Materials, vol. 25, no. 4, pp. 2122-2132, 2011. 

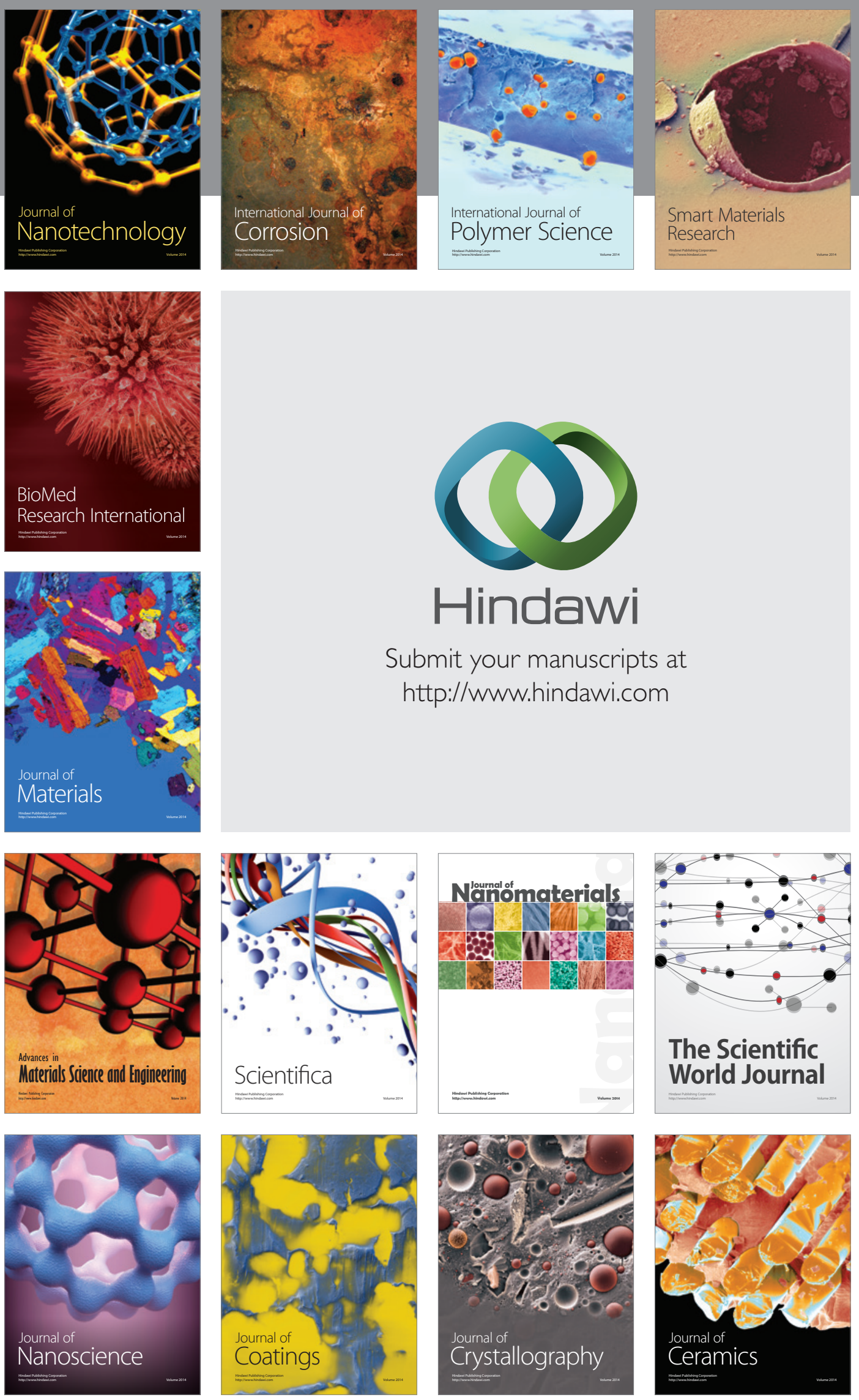

The Scientific World Journal

Submit your manuscripts at

http://www.hindawi.com

\section{World Journal}

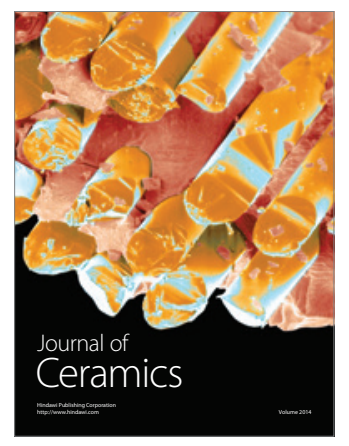

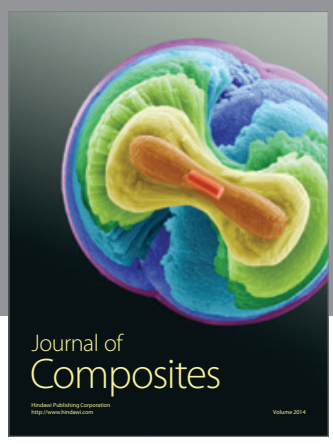
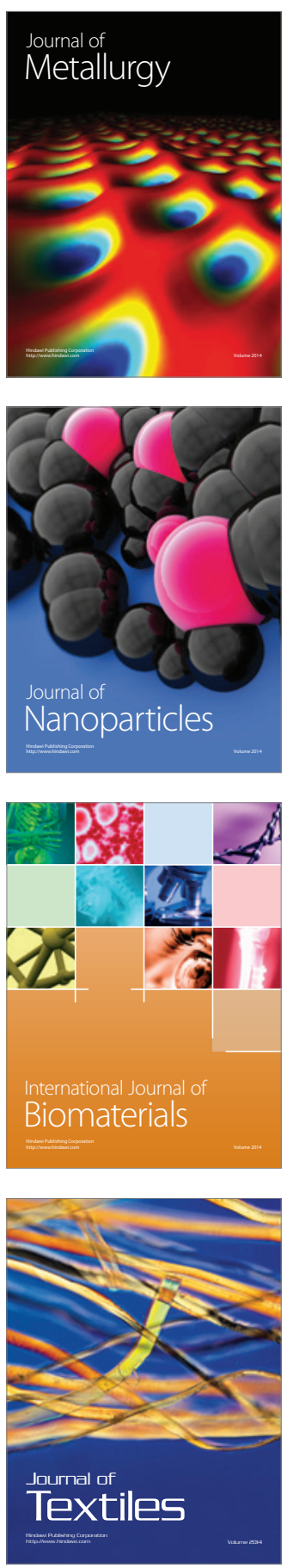